\title{
APPLYING THE TOPOLOGICAL DERIVATIVE AND THE BOUNDARY ELEMENT METHOD TO DESIGN OF SHAPE OF THE HEAT EXCHANGER
}

\author{
Katarzyna Freus ${ }^{1}$, Sebastian Freus ${ }^{2}$ \\ ${ }^{I}$ Institute of Mathematics, Czestochowa University of Technology \\ ${ }^{2}$ Institute of Computer and Information Science, Czestochowa University of Technology \\ Częstochowa, Poland \\ katarzyna.freus@im.pcz.pl,sebastian.freus@icis.pcz.pl
}

\begin{abstract}
In this work, the topological derivative for the Laplace equation is used to solve a design problem. This derivative describes the sensitivity of the problem when a small hole is formed at an arbitrary point of the domain. The goal of this work is to design topology of the domain when the Robin condition is imposed on the holes. Physically, the holes can be construed as cooling channels. For finding the solution of the governing equation the boundary element method is applied. The final part of the paper presents the design of the heat exchanger and results of computations.
\end{abstract}

Keywords: topological derivative, topological sensitivity, Laplace equation, boundary element method, heat transfer

\section{Introduction}

The topological derivative was first introduced by Céa et al. (1974). This one gives the sensitivity of a cost function when the domain under consideration is perturbed by the insertion of a hole. In this way, wherever this sensitivity is low enough (or high enough), depending on the problem, material is progressively removed. The topological derivative is often used for solving the topology design of several engineering problems (for example, design of heat conductors or heat exchanger) [1-3]. In this paper, we adopt a new approach called the topological-shape sensitivity method, which is based on classical shape sensitivity analysis. The topological-shape sensitivity method leads to a simple procedure to compute the topological derivative [1]. In this work, we firstly present a brief description of the topological derivative. Next, the problem is formulated and some details about the numerical method (BEM) are described. Finally, the design of a heat exchanger is shown. 


\section{A review of topological derivative}

In this work a topological derivative for the Laplace equation is applied. Let in the inside of the original domain $\Omega$ be formed a small hole of radius $\varepsilon$. The concept of the topological derivative $D_{T}^{*}$ is based on establishing the sensitivity of a given cost function (total potential energy) when the size of this hole is changed. The local value of the $D_{T}^{*}$ is obtained by [1-4]

$$
D_{T}^{*}(x)=\lim _{\varepsilon \rightarrow 0} \frac{\psi\left(\Omega_{\varepsilon}\right)-\psi(\Omega)}{f(\varepsilon)}
$$

where $\psi(\Omega)$ and $\psi\left(\Omega_{\varepsilon}\right)$ are the cost functions calculated for the original $\Omega$ and the new domain $\Omega_{\varepsilon}$, respectively, and $f(\varepsilon)$ is a regularizing function. The inconvenience of the definition (1) is the impossibility to determine a homeomorphism between domains with and without the hole. So, in [1] an alternative definition of the topological derivative called the topological-shape sensitivity method was proposed. The authors start from $\Omega_{\varepsilon}$, where the hole already exists, causing a small perturbation on the radius of the hole $\delta \varepsilon$ (see Fig. 1).

$$
D_{T}(x)=\lim _{\substack{\varepsilon \rightarrow 0 \\ \delta \varepsilon \rightarrow 0}} \frac{\psi\left(\Omega_{\varepsilon+\delta \varepsilon}\right)-\psi\left(\Omega_{\varepsilon}\right)}{f(\varepsilon+\delta \varepsilon)-f(\varepsilon)}
$$

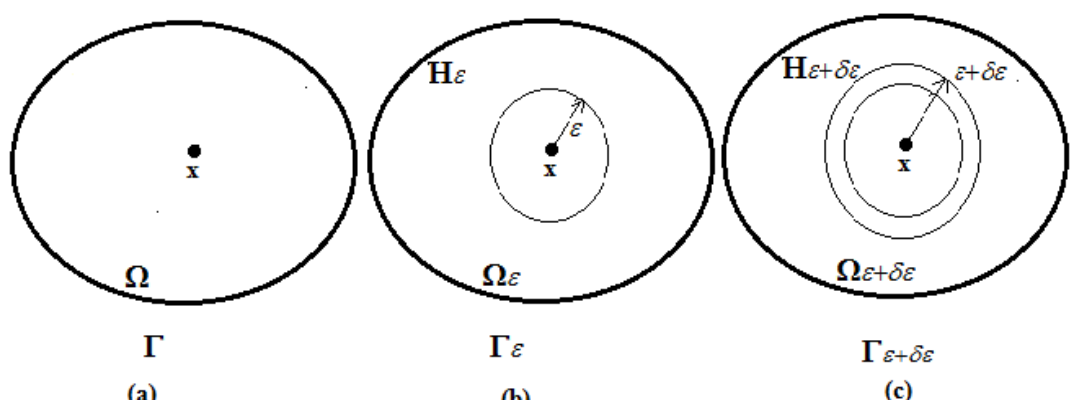

(a)

(b)

(c)

Fig. 1. Definition of the topological derivative: a) original concept, b), c) modified concept

Equations (1) and (2) are equivalent. However, the computation of $D_{T}$ is far easier than $D_{T}^{*}[1,2]$.

\section{Formulation of the problem}

In this paper the topological derivative is applied to a steady-state heat diffusion problem. The Laplace equation supplemented by the boundary conditions is taken into account $[1,4]$ 


$$
\begin{cases}x \in \Omega_{\varepsilon}: & \lambda \nabla^{2} T_{\varepsilon}(x)=0 \\ x \in \Gamma_{N}: & -\lambda \frac{\partial T_{\varepsilon}(x)}{\partial n}=q_{b} \\ x \in \Gamma_{R}: & -\lambda \frac{\partial T_{\varepsilon}(x)}{\partial n}=\alpha\left(T_{\varepsilon}(x)-T_{\infty}\right) \\ x \in \mathrm{H}_{\varepsilon}: & \lambda \frac{\partial T_{\varepsilon}(x)}{\partial n}+\alpha^{\varepsilon}\left(T_{\varepsilon}-T_{\infty}^{\varepsilon}\right)=0\end{cases}
$$

where $x=\left(x_{1}, x_{2}\right)$ are the spatial coordinates, $\lambda$ is the thermal conductivity, $T_{\varepsilon}(x)$ is the temperature, $\partial T_{\varepsilon} / \partial n$ denotes the normal derivative, $n=\left[\cos \alpha_{1}, \cos \alpha_{2}\right]$ is the normal outward vector, $q_{b}$ is the prescribed heat flux, $\alpha$ is the heat transfer coefficient and $T_{\infty}$ is the ambient temperature. On the holes $\mathrm{H}_{\varepsilon}$ the Robin boundary condition is prescribed where $\alpha^{\varepsilon}$ and $T_{\infty}^{\varepsilon}$ are the hole's internal convection parameters. In this case, the final expression for the topological derivative using the total potential energy as the cost function is the following:

$$
D_{T}(x)=-\frac{1}{2} \alpha^{\varepsilon} T\left(T-2 T_{\infty}^{\varepsilon}\right)
$$

Details of the calculation of $D_{T}$ are described in $[1,2]$.

It is important to mention that $T$ is the solution of the original problem (without a hole). In this work, the boundary element method was used to ensure the numerical solution.

\section{Numerical methodology}

In order to obtain the temperature field in the domain considered the boundary element method is used. The boundary integral equation for the Laplace equation is the following $[5,6]$ :

$$
\xi \in \Gamma: \quad B(\xi) T(\xi)+\int_{\Gamma} q(x) T^{*}(\xi, x) \mathrm{d} \Gamma=\int_{\Gamma} T(x) q^{*}(\xi, x) \mathrm{d} \Gamma
$$

where $B(\xi)$ is the coefficient from the interval $(0,1), \xi$ is the observation point, $T(x)$ and $q(x)=-\lambda \mathbf{n} \cdot \nabla T(x)$ are the temperature and the heat flux on the boundary $\Gamma$, respectively. For the problem considered, the functions $T^{*}$ and $q^{*}$ resulting from the fundamental solution are the following

$$
T^{*}(\xi, x)=\frac{1}{2 \pi \lambda} \ln \frac{1}{r}, \quad q^{*}(\xi, x)=\frac{d}{2 \pi r^{2}}
$$


where $r$ denotes the distance between $\xi=\left(\xi_{1}, \xi_{2}\right)$ and $x=\left(x_{1}, x_{2}\right)$

$$
r=\sqrt{\left(x_{1}-\xi_{1}\right)^{2}+\left(x_{2}-\xi_{2}\right)^{2}}
$$

while

$$
d=\left(x_{1}-\xi_{1}\right) n_{x}+\left(x_{2}-\xi_{2}\right) n_{y}
$$

$n_{x}, n_{y}$ are the directional cosines of the normal outward vector $\mathbf{n}$.

In order to solve equation (5) the boundary is divided into $N$ linear boundary elements. The integrals in equation (5) are substituted by the sums of integrals over these elements

$$
B\left(\xi_{i}\right) T\left(\xi_{i}\right)+\sum_{j=1}^{N} \int_{\Gamma_{j}} q(x) T^{*}\left(\xi_{i}, x\right) \mathrm{d} \Gamma_{j}=\sum_{j=1}^{N} \int_{\Gamma_{j}} T(x) q^{*}\left(\xi_{i}, x\right) \mathrm{d} \Gamma_{j}
$$

Next, one obtains the following system of algebraic equations

$$
\sum_{r=1}^{R} G_{i r} q_{r}=\sum_{r=1}^{R} H_{i r} T_{r}, \quad i=1,2, \ldots, R
$$

This system of equations can be written in the form

$$
\mathbf{G q}=\mathbf{H T}
$$

Taking into account the known boundary conditions, equation (11) can be reordered

$$
\mathbf{A X}=\mathbf{B}
$$

where $\mathbf{A}$ is the main matrix, $\mathbf{X}$ is the unknown vector and $\mathbf{B}$ is the free terms vector. Solving equation (12), all the boundary variables are known.

The temperature in the interior nodes can be calculated using the following formula

$$
\xi \in \Omega: \quad T(\xi)=\int_{\Gamma} T(x) q^{*}(\xi, x) \mathrm{d} \Gamma-\int_{\Gamma} q(x) T^{*}(\xi, x) \mathrm{d} \Gamma
$$

or

$$
T\left(\xi^{i}\right)=\sum_{r=1}^{R} H_{i r} T_{r}-\sum_{r=1}^{R} G_{i r} q_{r}
$$

Details about the BEM can be found in $[5,6]$. 


\section{Numerical example}

In this part of paper the design of a heat exchanger is presented. The rectangular domain of dimensions $2 L \times L(L=2 \mathrm{~m})$ shown in Figure 2 has been considered. The hatched areas of width $h=0.5 \mathrm{~m}$ each (see Fig. 2) will not be perturbed (this is the structural part of the problem). Thermal conductivity equals $\lambda=330 \mathrm{~W} /(\mathrm{mK})$. On the top of the domain the Robin condition is taken into account where the heat transfer coefficient is $\alpha=20 \mathrm{~W} /\left(\mathrm{m}^{2} \mathrm{~K}\right)$ and the ambient temperature is $T_{\infty}=25^{\circ} \mathrm{C}$. On the bottom of the rectangular, the heat flux presents a piecewise linear distribution where the smallest value is $q_{b 1}=2000 \mathrm{~W} / \mathrm{m}^{2}$ and the greatest value is $q_{b 2}=20000 \mathrm{~W} / \mathrm{m}^{2}$, on the remaining parts of the boundary the Neumann condition $q_{b}=0$ is prescribed. The initial boundary has been divided into 90 linear boundary elements. The grid of 450 internal nodes has been used.

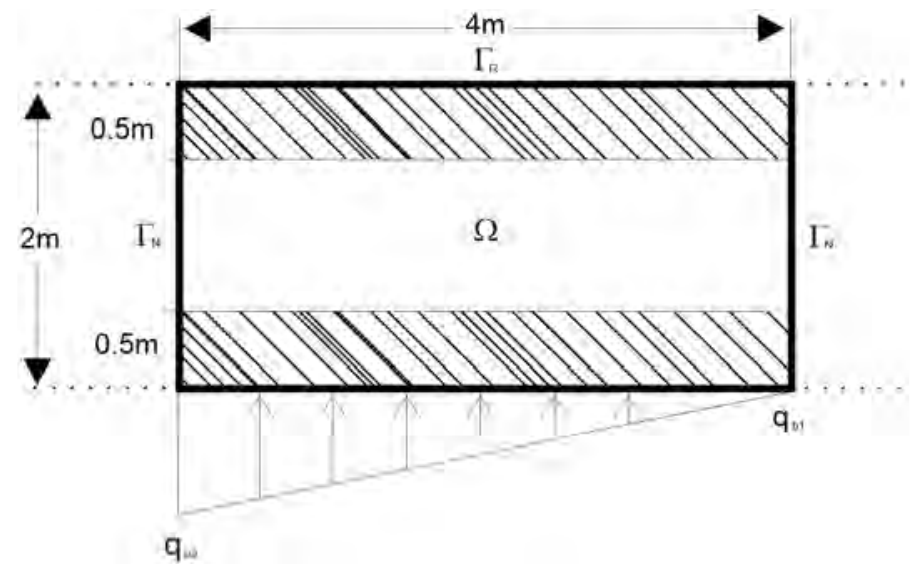

Fig. 2. Domain considered

On the holes created via topological derivative, the Robin condition is prescribed where $\alpha^{\varepsilon}=200 \mathrm{~W} /\left(\mathrm{m}^{2} \mathrm{~K}\right)$ and $T_{\infty}^{\varepsilon}=30^{\circ} \mathrm{C}$. Hence $D_{T}$ will be evaluated using the formula (see (4))

$$
D_{T}(x)=-\frac{1}{2} \alpha^{\varepsilon} T\left(T-2 T_{\infty}^{\varepsilon}\right)
$$

In order to obtain the design of the domain, the following iterative process is carried out in 7 steps:

Step 1. Provide the initial domain

Step 2. Solve the problem using the BEM

Step 3. Calculate $D_{T}$ at internal points (using Eq. (15))

Step 4. Select the point with the greatest absolute values of $D_{T}$

Step 5. On the selected point create a hole

Step 6. Check the stop criterion

Step 7. Repeat the procedure until a given stopping criteria is obtained 
The iterative process is stopped if the maximum temperature of domain $\Omega$ decreases below $170^{\circ} \mathrm{C}$. During each iteration, $2.5 \%$ of material is eliminated. The boundary of the holes has been divided into 6 linear boundary elements (see Fig. 3). The temperature distribution obtained during the iterative process is presented in Figures 4, 6, 8, 10,12 whereas Figures 5, 7, 9, 11 and 13 show the topological derivative received during the iterative process.

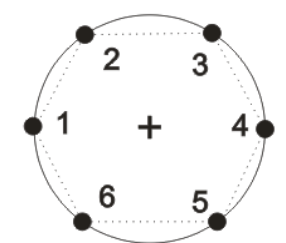

Fig. 3. Hexagonal hole

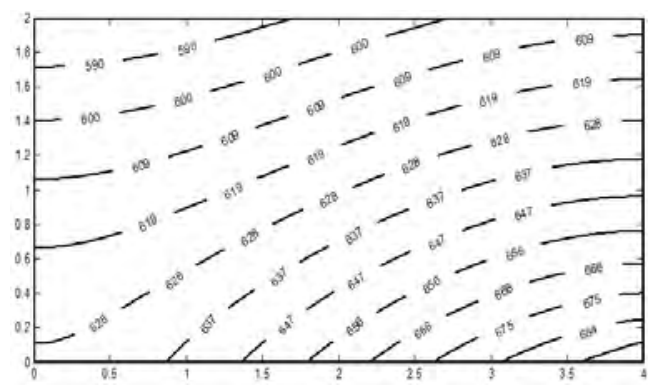

Fig. 4. Temperature distribution at $i=0$

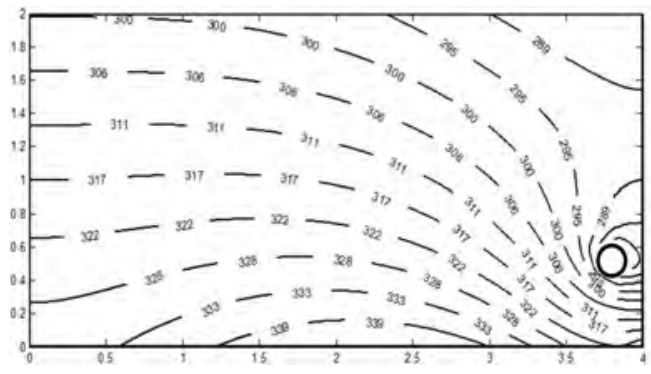

Fig. 6. Temperature distribution at $i=1$

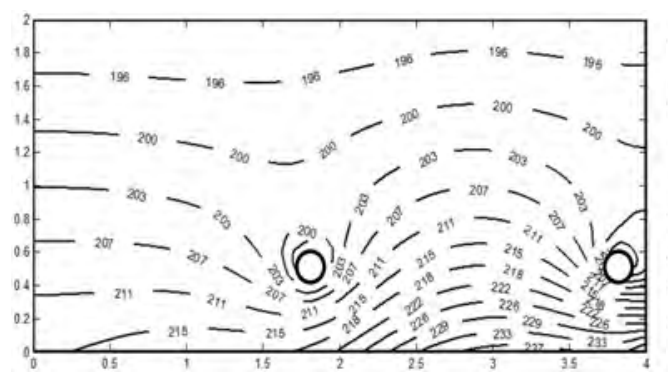

Fig. 8. Temperature distribution at $i=2$

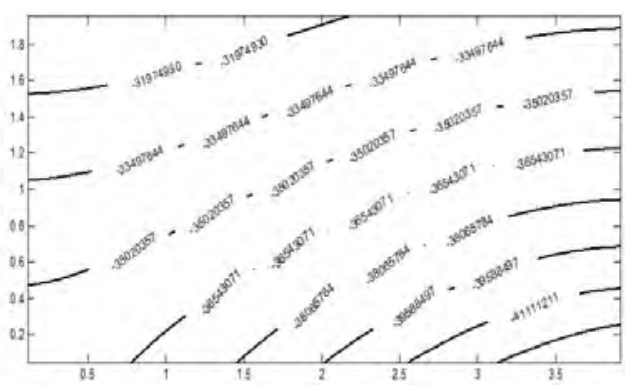

Fig. 5. Topological derivative at $i=0$

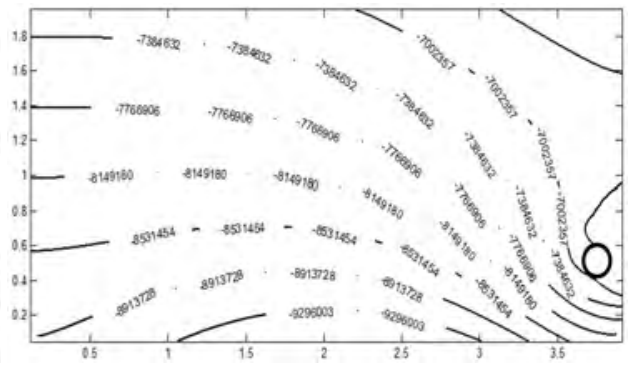

Fig. 7. Topological derivative at $i=1$

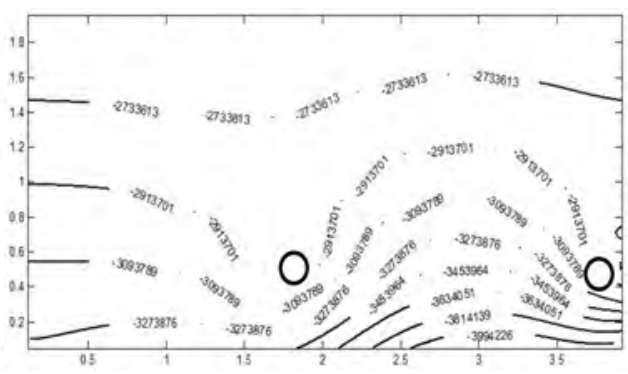

Fig. 9. Topological derivative at $i=2$ 


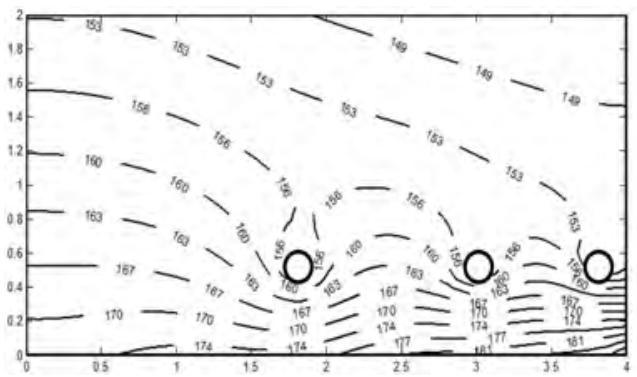

Fig. 10. Temperature distribution at $i=3$

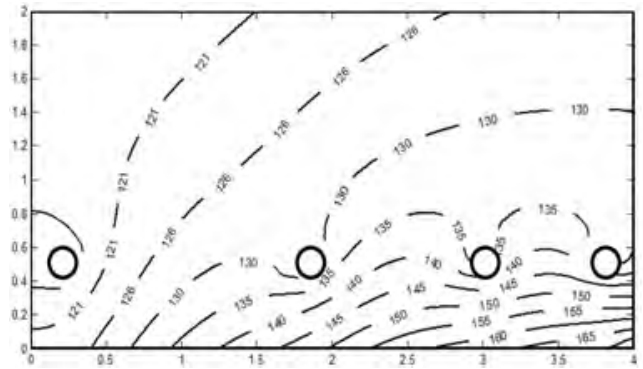

Fig. 12. Temperature distribution at $i=4$

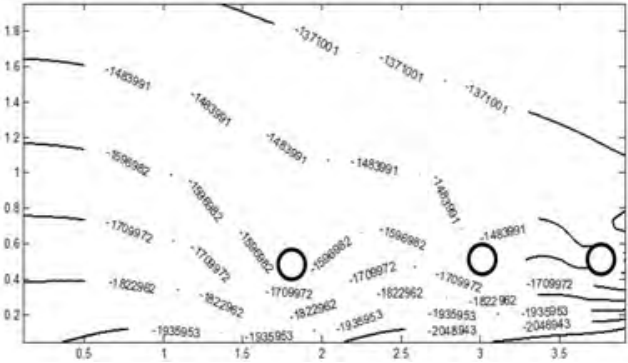

Fig. 11. Topological derivative at $i=3$

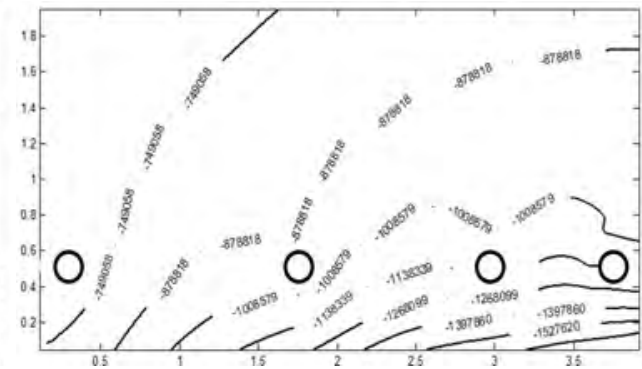

Fig. 13. Topological derivative at $i=4$

This example shows how the topological derivative can be used to design heat exchangers in order to determinate where the cooling channels (holes) must be positioned. It is worth noting, that the distance between the holes gradually grows. The final result was obtained at iteration $i=4$, as can be seen in Figures 12 and 13 . This result was compared with a similar problem available in the literature [1] that was calculated by means of a FEM. In both cases the final designs are close.

\section{Conclusions}

In the present work the topological derivative is used to obtain the project of the heat exchanger. The topological-shape sensitivity method gives information concerning the positions where the holes (cooling channels) must be created. The temperature field in each iteration is calculated by means of the boundary element method in its direct version. The connection of the topological derivative and the BEM is a good tool that can be applied for solving design engineering problems in heat transfer.

\section{References}

[1] Navotny A.A., Feijoo R.A., Taroco E., Padra C., Topological-shape sensitivity analysis, Comput. Methods Appl. Mech. Eng. 2003, 192, 803-829.

[2] Marczak R.J., Topology optimization and boundary elements - a preliminary implementation for linear heat transfer, Engineering Analysis with Boundary Elements 2007, 31, 793-802. 
[3] Anflor C.T.M., Marczak R.J., Topological sensitivity analysis for two-dimensional heat transfer problems using the Boundary Element Method, Optimization of Structures and Components Advanced Structured Materials 2013, 43, 11-33.

[4] Freus K., Freus S., Determination of an optimal shape of domain using the topological derivative and Boundary Element Method, Journal of Applied Mathematics and Computational Mechanics 2014, 13(4), 41-48.

[5] Brebbia C.A., Dominguez J., Boundary Elements. An Introductory Course, CMP, McGraw-Hill Book Company, London 1992.

[6] Majchrzak E., Boundary Element Method in Heat Transfer, Publ. of Czestochowa University of Technology, Czestochowa 2001 (in Polish). 